\title{
Development of Location Detection and Human Tracking Application via GPS and GSM Service: A Pilot Study
}

\author{
Inurina Ibrahim and Engku Mohammad Nazreen B. Engku Azman
}

\begin{abstract}
With the development and awareness of social affairs concerning locating missing person and location detection services has been widely on demand. This paper explains the design and experiment of incorporating the GPS \& GSM network positioning technology to deliver a locationbased service for tracking and detecting human. In this study, GPS and GSM feature were utilized to emulate the tracking and detection process. Integration of this technology is supported by the use of several applications, devices and services. The proposed model comprises of GPS sender and receiver, GSM service and provider. With the extensive use of smartphones and mobile applications, this model is useful to identify the location of a person in a public area and may benefit parents in monitoring and tracking their children whereabouts. This paper, presented the methods adopted in developing of the initial prototype as a pilot study. The result of the evaluation discussed recommendations for further improvement in the prototype development. This shall be achieved by adding specific values for a better outcome. A detail result on the improvement will be discussed in the next paper. The purpose of this study is to share this information with other researcher and to foresee ways to improve the current processes hence the same study can be initiated and explored by others. A further enhancement of this service shall be proposed and another set of evaluation should be performed thus there be a potential for application commercialization.
\end{abstract}

Index Terms-Global positioning system (GPS), global system for mobile (GSM), human tracking and detection.

\section{INTRODUCTION}

Many cases of missing person in a public area were reported and fast detection is highly demanded. This situation may be commonly seen in the after effect of natural disasters, earth quake, tsunami and others. Other scenario where people is always on the move, for instance annual Hajj seasons where there are too many people move in groups from one location to another may leads to tendency of missing a group members including spouse, siblings or friends. Thus getting hold of one person may be difficult to be performed without the aid of automated process or tools. While missing person case is another popular daily newspaper storyline. This situation occurs all over the globe. Hence with the Social responsibility and awareness of the community concerning this issue, a study on developing the service model via GPS and GSM technology were conducted. The huge population of smartphones users all over the world is the reason being why these services is adopted in this

Manuscript received May 18, 2015; revised September 16, 2016.

The authors are with the Software Information Systems Section at Universiti Kuala Lumpur, Malaysian Institute of Information Technology, Malaysia (e-mail: lnurina@unikl.edu.my, emnazreeneaman@gmail.com). study. Generally in this study will involve the use of smartphones and the telecommunication services. With the Global Positioning System (GPS) as a medium using the satellite-based navigation system, it will provide the latitude $\&$ longitude of the device. This system comprise of a database of every registered routes and location recorded on the map. The Global System for Mobile (GSM) technology is adopted to transmit voice and text to complement the proposed prototype, this is to for the purpose of supporting emergency cases. GSM service is widely used as communication medium where sending and receiving process occur via a device from remote locations [1], [2].

\section{What IS HUMAN TRACKING AND DETECTION}

The Human Tracking and Detection service comprise of combination of a devices and application to perform the tracking and detecting process. Two devices are required to achieve this. Device A will is use to send signals, this is known as the tracking device. While device $\mathrm{B}$ is used as receiver consists of an android smartphone. The detail requirement of each of the devices is as follows:

i. Human Tracking and Detection device (Device A)

a. Sending the coordinate

b. Sending panic alert with Real-time voice message

\section{ii. Smartphone user (Device B)}

a. View coordinates via Location Identification Application

b. Receiver can receive coordinate

c. Receiver can receive Real-time voice message

d. Receiver can send message request to the Sender device

\section{A. Why Human Tracking and Detection}

Identifying and locating a person in an infinite public area is not easy. What more if it is done manually. This may be the after effect situation where the occurrence of natural disaster such as earthquake, huge flood and others. According to reports in Malaysian tabloids, on average there are four (4) missing person daily reported. Many of the missing persons identified were aged below eighteen (18) years old [3].

Almost every day, cases of missing person were reported. A gadget to prevent this is needed thus a person's location can be tracked and detected. In emergency cases where phone call is impossible, a device may save life. Many have been looking for solution as a service to inhibit situation. With this issue to solve, with the widely use existing GPS \& GSM technology, a pilot study is conducted to experiment 
the development of a human tracking and detection application prototype.

\section{RELATED WORK}

Many GPS and GSM technology models have been developed to trace mobility of an object such as vehicle, person or anything physically detectable. Several related studies is as highlighted [1].

A cost effective method of object tracking using GPS and GPRS technology was proposed by Khondker Shajadul Hasan, Mashiur Rahman, Abdul L. Haque, M. Abdur Rahman, Tanzil Rahman and M. Mahbubur Rashed in 2009. This method allows user to view the location of the object as well as the past history of its movement using Google Map and Internet [4].

In 2011, Ruchika Gupta in "GPS and GPRS Based Cost Effective Human Tracking System Using Mobile Phones" proposed a mobile phone application developed and deployed on an Android Phone to track the GPS location and send it to a remote location by creating a GPRS packet. This is an approach that the whole system allows the user's mobility to be tracked using a mobile phone which is equipped with an internal GPS receiver and GPRS transmitter [5].

\section{A. $(G S M)$}

Global Positioning System (GSM).The use of GSM gained its popularity by more than 200 countries in the world since early 2000 and by 2005 this technology has been subscribed by more than 1.5 people which represents $78 \%$ of the world market [6]. Based on the result of the study, Sohn et al. on Mobility detection using GSM traces, proved that GSM sensor is best used to detect high level activity on mobile phones in any location. Hence this sensor is adopted in this study to suit the purpose.

Fig. 1 below shows the result of GSM sensor based on study performed by Sohn et al. regarding precision and predicted movement. The overall result shows $85 \%$ accuracy of the tests.

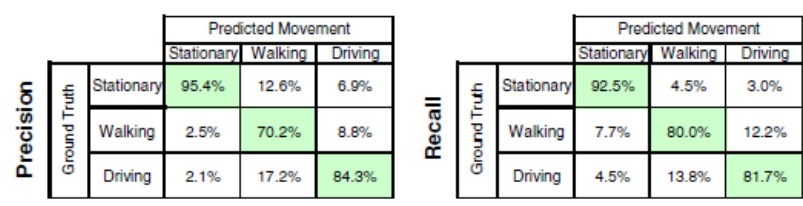

Fig. 1. Result of GSM sensor based on study performed by Sohn et al.

Sameer Darekar, Atul Chikane, Rutujit Diwate, Amol Deshmukh, and Prof. Archana Shinde in 2012, have developed a Multi-Tracking system to trace objects using GPS and GSM technology. They have recommended many other areas of study to be conducted such as tracking movable assets, antitheft vehicle and managing transportation may be applied in the future [7].

In 2013, Anjor Jadhav, Savita Kharje, Pooja More and Prof. Nasim Shah proposed a mobile tracking application based on GPS using the geographical co-ordinates of the user to locate the mobile device. It claims able to effectively calculate the distance between the user and the located friends based on the ones who are closest to the user. The
GSM service is used by sending SMS to the selected mobile number to notify message and location of the person [8].

\section{THE Proposed SERVICE MOdEL}

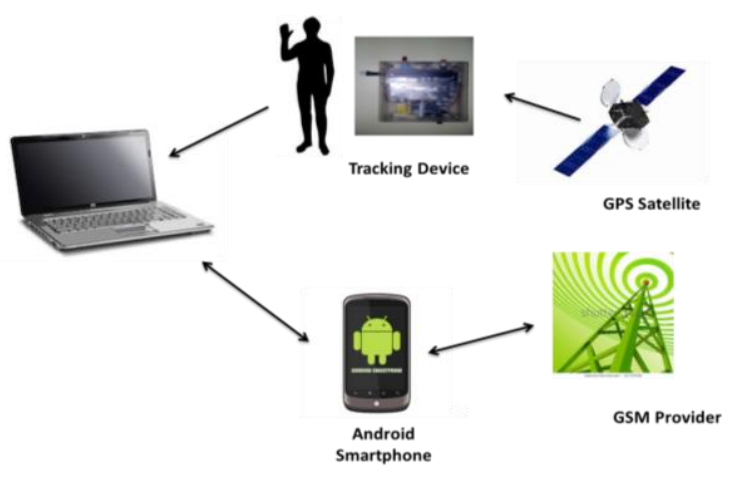

Fig. 2. The system architecture.

For this service to work, a wireless internet connection is required for both the tracking and mobile phone devices. The tracking device will set on the LED to indicate a start (ON and OFF) indicator in approximately ten (10) seconds. Coordinate is then sent to the specific smartphone as the receiver via a service provider server. The receiver then will receive an SMS from the tracking device with a specific alert message and attached the link to the coordinate of the senders' location. The receiver on smartphone then will be able to click on the link and retrieve the location via a map application which gets the information from the GPS database service. The tracking device also transmits the voice signal to provide detail situation from the senders' device (see Fig. 2).

\section{A. The Physical Components}

To run this research, investments on hardware, software and service providers is required. The detail components required as shown in Table $\mathrm{I}$.

\begin{tabular}{|c|c|}
\hline Hardware & Software \\
\hline Tracking device A & Arduino C \\
\hline $\begin{array}{l}\text { i. Micro Controller : Arduino } \\
\text { Uno ATmega328 GSM } \\
\text { Module }\end{array}$ & \\
\hline Arduino Shield GSM & \\
\hline GPS Module : EM406A & \\
\hline Mini Microphone & \\
\hline ON/OFF button & \\
\hline Mobile phone & $\begin{array}{l}\text { Short Messaging Service } \\
\text { SMS }\end{array}$ \\
\hline
\end{tabular}

\section{METHODOLOGY}

To achieve the development of this service, the Spiral model is adopted. This combines the idea of iterative development with the systematic and controlled aspects of the waterfall model. It involves incremental releases of the product, or incremental refinement through each of iteration around the spiral. Hence testing and refining is concurrently on-going. 
The process includes, initial study, design, development and evaluation which is performed iteratively along the pilot study. This method has been applied by many researcher and system developer with proven success.

\section{A. The Prototype}

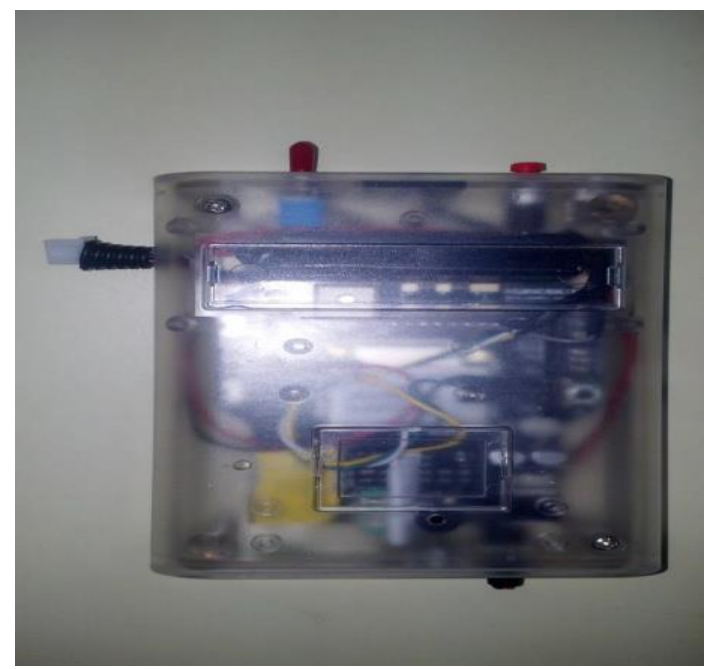

Fig. 3. Device A: tracking device.

To develop the tracking device (see Fig. 3) a microcontroller board, Arduino Uno AT model is customized. The GPS and GSM module is embedded onto the board. This is to enable the tracking and detection to occur. An additional ON/OFF button is embedded to the board to trigger the start and end of alert message from the device. In addition a mini microphone was attached to the board, this is to allow the voice data transmitted to the receiver [9], [10].

In this experiment, Arduino board is selected due to its flexibility in transmitting analog and digital signal. It allows connection to a personal computer via USB and it is able to run on a stand-alone mode. A range of several programming languages is allowed to enable the communication process with other devices. Overall this model is cost effective to run the pilot study [2], [11].

\section{B. Receiving Device}

Another device to run this service model is a mobile phone. For the purpose of this study, the mobile phone model used is Samsung Galaxy A8. The communication service subscribed on this mobile phone is DiGi. The phone should be equipped with a Wi-Fi connection by a service provider. This receiving device will be detecting signals sent from the tracking device. Data sent includes the coordinate location of the senders' device and an alert message defaulted set from the senders end. In addition the coordinate was sent via a URL link to the Google applications map received via a Short Messaging Service (SMS). Besides the coordinate and text message, the senders device also allows the transmission of voice data. This is transmitted by an installed microphone component on the Arduino board. This allows the default mobile phone capabilities to receive voice data. The purpose of this is to help receiver to judge if the situation from the senders device if it is genuine or otherwise. This is really helpful when emergency condition occurs. Refer outcome of the data received in Fig. 4 and Fig. 5.

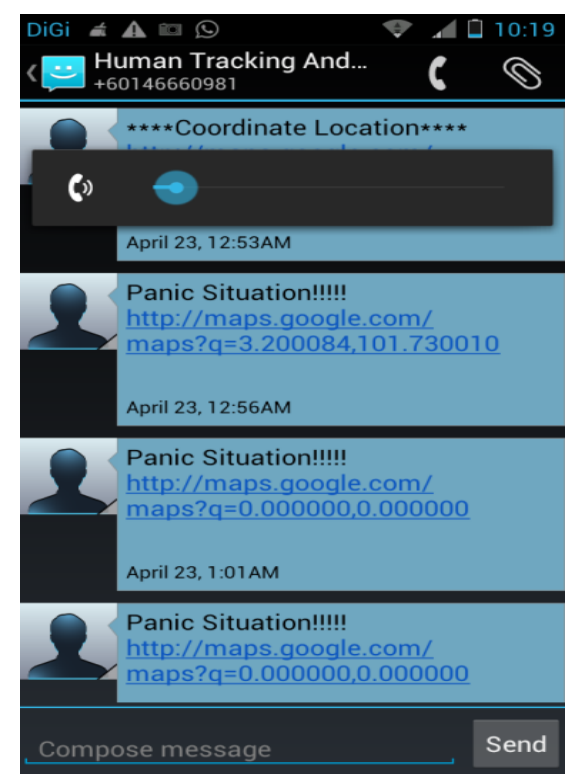

Fig. 4. Device B: Mobile phone.

Fig. 4 illustrates the application from the sample mobile phone. It shows a situation when a signal being received providing a link to the coordinate from the tracking device. Once the link to the location is clicked, the system will then redirect for options to launch the application to the map as shown in Fig. 5.

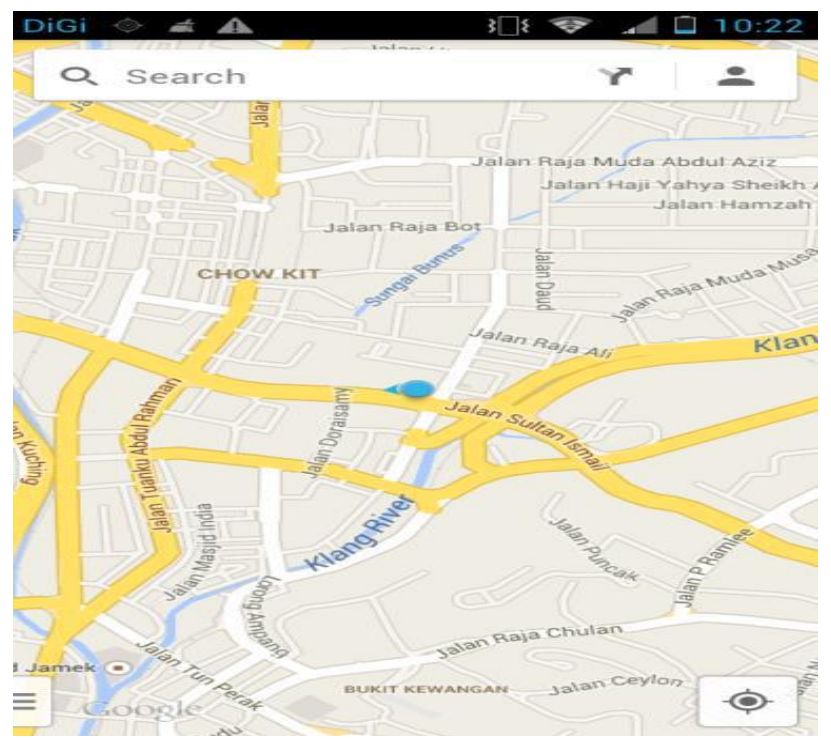

Fig. 5. Mobile phone: location on map API

\section{EVALUATION}

At the point of this writing, the human tracking and detecting model has gone through the pilot testing stage. For the purpose of this testing, the developed prototype is put on an integration tests. This is to test Device A : Senders Device (Customized Arduino Board) on its success to communicate to Device B : Receiving Device (Mobile Phone) Based on the project planned and the proposed design the following are the features to be tested:

i. Accuracy of the GPS system in getting coordinate

ii. Availability of the GSM system to sending report to the user 
iii. Efficiency of the GPS and GSM to receive and sending data.

To achieve the evaluation phase, several tests was planned. In this pilot study the integration test was successfully performed and the result was recorded. This test was run by the developer evaluate the working of each module and its integration on devices and application. The detail result of the pilot test is as shown in Table II.

TABLE II: RESULT OF PILOT TEST

\begin{tabular}{|l|l|l|c|}
\hline No & Source of Test & Result & $\begin{array}{l}\text { Sample } \\
\text { Tests }\end{array}$ \\
\hline 1 & Accuracy of coordinates & $\begin{array}{l}4 / 5 \text { results showed } \\
\text { accurate } \\
\text { coordinates }\end{array}$ & 5 \\
\hline 2 & 1 Minute Delay & $\begin{array}{l}3 / 5 \text { results showed } \\
\text { delay time exists } \\
\text { (unknown reason) }\end{array}$ & 5 \\
\hline 3 & Message delay highly & $\begin{array}{l}3 / 5 \text { results show that } \\
\text { message delay due to } \\
\text { the telecommunication } \\
\text { provider }\end{array}$ & \\
\hline
\end{tabular}

Corrective actions were iteratively performed to achieve the modules objective during the pilot testing.

The number of result samples collected for the test is five (5). The first test is the accuracy of the coordinates. Four (4) out of five (5), or eighty percent $(80 \%)$ of the test concludes the coordinated read is highly accurate based on the google map. The second source is tesing if there is delay within the range of one minute of time for message sending and receiving. The result shows three (3) out of five (5), or sixty percent $(60 \%)$ of the result proves that delay on message relay exists this in with unknown reason. While the final source tested for message delay due to service provider shows that three (3) our of five (5) or $(60 \%)$ is due to service provider's fault.

In the Pilot test, the type of mobile devices used is a Samsung Galaxy A8 Model, and the mobile telecommunication carrier subsribed was Digi. The result however did not perform a test on other mobile phone model and telecommunication services. In the future this shall be consider as it may show a variation of result if it is well specified. Even though the timing of message sending and recieving was recorded, the results recorded may depend on the telecommunication providers. A further investigation and improvement shall be conducted to specify the relationship between the service provider and the delay in receiving the message over the communication line.

The plan for the next phase of testing is the functionality test. This phase of testing will involve validating the application and specifications to correctly perform all its required functions. The set of test will be executed when the units of the application are considered complete. It will involve the operations and back-end testing to access each level of functionality including the overall service characteristic, the performance and the accuracy. This test phase is one of the way to determine the prototype developed is functioning as intended.

\section{A. Future Enhancement}

Based on the pilot study performed, few areas showed the need for improvements and further investigations. The possible contributors to the results is elaborate as the followings.

The first is the type of device used, as there are various types mobile phones available in the market. This mobile phone types act as receiver may reflect the test result, hence in the future the type and model of the mobile device should be clearly identified and tested. The second is the telecommunication carrier. This may augment the result as there are many different telecommunication carrier available in the country. While different telecommunication link may lead to different readings during evaluation. The third source tested is the range of the distance between the senders' and the receiving device. This is to evaluate if the location of the sender and receiver distance if it effects the overall result. For this pilot study the distance involved during the testing is approximately 1 kilometer. In the future a different range of distance should be tested to identify if there is an impact to the result of the test.

The fourth variable proposed to be added in the test is the duration of the sending and receiving of data. For the purpose of this pilot study, the duration only monitors a delay in a range of one (1) minute for the data to be successfully transmitted. Thus in the future the actual timing on the successfull data transmitted shall be recorded accordingly. Same goes to the variance of the distance.

Based on the result of the pilot testing, an improvement on the development and further testing of the current model should be planned. This shall include finding the possibility of incorporating the GPRS services and android development to add more flexibility to the current model [12], [13]. Another step on further enhancement is required if this model is to be commercialized. Hence User Acceptance Testing should be performed. A User Acceptance Testing (UAT) is a process to obtain confirmation that a system meets mutually agreed-upon requirements. A Subject Matter Expert (SME), preferably the owner or client of the object under test, provides such confirmation after trial or review. In software development, UAT is one of the final stages of a project and often occurs before a client accepts the new system.

\section{REFERENCES}

[1] A. S. Dinkar et al., "Design and implementation of vehicle tracking system using GPS," Journal of Information Engineering and Applications, vol. 1, no. 3, 2011.

[2] P. Kumar and P. Kumar, "Arduino based wireless intrusion detection using IR sensor and GSM," IJCSMC, vol. 2, issue 5, pp. 417-424, May 2013.

[3] S. Saari, Wan Noor Hayati Wan Alias. Laporan Khas. Purata 4 Hilang Setiap Hari, Utusan Malaysia, 2011.

[4] K. S. Hasan, M. Rahman, A. L. Haque, M. A. Rahman, T. Rahman, and M. M. Rasheed, "Cost effective GPS-GPRS based object tracking system," in Proc. the International Multi-Conference of Engineers and Computer Scientists, March 18-20, 2009, Hong Kong.

[5] R. Gupta and B. Reddy, "GPS and GPRS based cost effective human tracking system using mobile phones," Viewpoint, vol. 2, no. 1, January-June 2011.

[6] T. Sohn, A. Varshavsky, A. LaMarca, M. Y. Chen, T. Choudhury, I. Smith, S. Consolvo, J. Hightower, W. G. Griswold, and E. de Lara. Mobility Detection Using Everyday GSM Trace. 
[7] S. Darekar, A. Chikane, R. Diwate, A. Deshmukh, A. Shinde, "Tracking system using GPS and GSM: Practical approach," International Journal of Scientific \& Engineering Research, vol. 3, issue 5, May-2012.

[8] A. Jadhav, S. Kharje, P. More, and N. Shah, "Track your buddies," in Proc. National Conference on New Horizons in IT, 2013.

[9] P. Teikaria, R. P. Najjara, and H. Malkkic, "An inexpensive Arduinobased LED stimulator system for vision research," Elsevier Journal of Neuroscience Methods, vol. 211, issue 2, pp. 227-236, November 15, 2012.

[10] M. Bohmer, Beginning Android ADK with Adruino, APress.

[11] A. Garg, A. K. Shudhanshu, R. Kumar, S. R. Bharadwaj, R. Tehri, and C. K. Samal, "Development of open source low cost wireless data acquisition systems for science experiments," International Journal of Information \& Computation Technology, vol. 4, no. 12, pp. 11231128, 2014.

[12] S. Lee, G. Tewolde, and J. Kwon, "Design and Implementation of Vehicle Tracking System Using GPS/GSM/GPRS Technology and Smartphone Application," in Proc. 2014 IEEE World Forum on Internet of Things (WF-IoT), IEEE.

[13] K. Maurya, M. Singh, and N. Jain, "Real time vehicle tracking system using GSM and GPS technology - An anti-theft tracking system," International Journal of Electronics and Computer Science Engineering.

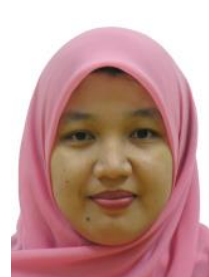

Inurina Ibrahim was born in Kuala Lumpur Malaysia on 19 May 1974. Graduated in Bachelor of Science in Business Administration Majoring in Management Science and Information Systems from University of Rhode Island, USA in 1997. Completed her MSc (Hons) in Information Technology from Universit Utara Malaysia in 2007

She has been in the academic field for 18 years since the 1998. Before joining as academic members, she had experience working with the service industry as the Web Master and IT Executive. During her employment with University Kuala Lumpur, she gained good numbers of experienced in the area of System Development \& Design as well as Project Management. Experienced to work on SUTRA Project as a Consultant and Team Leader in a SUTRA Project in 2012 to 2013.

Inurina Ibrahim is a Certified EC-Council E Commerce Professional in Project Management. She have published numbers of research papers including one on Teaching Project Management Methods and Approach for IT Students. 